\title{
Low Beta MHD Equilibrium Including a Static Magnetic Island for Reduced MHD Equations in a Straight Heliotron Configuration
}

\author{
Kinya SAITO ${ }^{1, a)}$, Katsuji ICHIGUCHI ${ }^{1,2)}$ and Ryuichi ISHIZAKI ${ }^{1,2)}$ \\ 1) The Graduate University for Advanced Studies, Toki, Gifu 509-5292, Japan \\ 2) National Institute for Fusion Science, Toki, Gifu 509-5292, Japan
}

(Received 6 December 2011 / Accepted 18 April 2012)

\begin{abstract}
Low beta MHD equilibria including static magnetic islands generated by an external field are studied by using a two-step approach. The equilibria correspond to the reduced MHD equations in a straight heliotron configuration. In the first step, a diffusion equation parallel to the field line is solved with the magnetic field fixed for a solution of the pressure constant along field lines. In the second step, the equilibrium equation corresponding to the vorticity equation is solved with the pressure fixed for a solution of the poloidal magnetic flux. The two steps are iterated until the width of the island is converged. The equilibrium pressure profile as a result of the method is locally flat at both the O-point and the X-point of the magnetic island. Effects of a pressure diffusion perpendicular to the field are also studied. In this case, an equilibrium pressure of which the profile is flat at the $\mathrm{O}$-point and steep at the X-point is obtained.
\end{abstract}

(c) 2012 The Japan Society of Plasma Science and Nuclear Fusion Research

Keywords: static magnetic island, reduced MHD equation, straight heliotron configuration, equilibrium pressure profile, diffusion perpendicular to the field

DOI: $10.1585 /$ pfr.7.1403070

\section{Introduction}

In magnetic configurations of heliotron devices such as the Large Helical Device (LHD) [1], a magnetic hill usually exists in the plasma confinement region. Therefore, resistive interchange modes driven by pressure gradients are easily destabilized in the heliotron devices. Since the interchange modes can cause a collapse of the plasma, the stability control is crucial for a good confinement at high beta.

On the other hand, nested magnetic surfaces are also desirable for the good confinement. However, error magnetic fields originated from coil misalignment and the terrestrial magnetism induce static magnetic islands. Such magnetic islands have a possibility to degrade plasma confinement. On the other hand, magnetic islands are also generated by the nonlinear saturation of the interchange modes [2,3]. Therefore, the static magnetic islands have a potential to affect the stability of interchange modes, that is, the magnetic islands and the interchange modes can interact with each other.

In our original work $[4,5]$, the interaction between static magnetic islands and interchange modes with the same mode number in the cylindrical geometry is numerically studied. Especially, we focus on a magnetic island with the mode number of $(m, n)=(1,1)$, where $m$ and $n$

\footnotetext{
author'se-mail: saito@nufd.jp

a) present address: Numerical Flow Designing, CO. LTD., Shinagawaku, Tokyo 141-0022, Japan
}

are the poloidal and toroidal mode numbers, respectively. The reduced MHD equations [6] are utilized for the analysis because it is useful for the analysis of such low mode number interaction. A straight heliotron configuration with a magnetic hill is examined for the investigation of the basic mechanism. A pressure profile corresponding to the nested magnetic surfaces is used for the equilibrium pressure. In this case, the interchange modes grow as in the case without the static magnetic island. The width of the magnetic island is changed by the nonlinear saturation of the interchange mode. The situation of the increase or decrease of the width depends on whether the diffusion of the equilibrium pressure in the direction parallel to the magnetic field is taken into account or not. In the case without the parallel diffusion of the equilibrium pressure, there exist two solutions corresponding to the increase and the decrease of the island width [4]. In the case with the parallel diffusion of the equilibrium pressure, there exists only one solution corresponding to the increase of the width [5].

The growth of the interchange modes in the above studies is attributed to the fact that the pressure profile corresponding to nested surfaces is employed as the equilibrium pressure. The pressure profile has finite gradient inside the separatrix, which makes the interchange mode grow. Other works [7-9], which treated the static islands and the interchange modes, also employed pressure profiles corresponding to the nested surfaces as the equilibrium pressure. This kind of profile does not correspond to 
the magnetic surfaces with the magnetic islands. Therefore, the effect of the magnetic islands on the equilibrium pressure profiles is not included. Generally, the equilibrium pressure is considered to be changed due to the existence of the magnetic islands. Therefore, the equilibrium pressure profile including magnetic islands has possibility to affect the stability of the interchange modes. Final goal of our research is to study how the static islands changes the stability. For this study, an equilibrium with the pressure consistent with static magnetic islands is required. Thus, in this work, we develop a numerical code to obtain MHD equilibria consistent with the static magnetic island with the mode numbers of $(m, n)=(1,1)$, which correspond to the reduced MHD equations in a straight heliotron configuration.

The development of numerical codes to solve an equilibrium including magnetic islands has a long history. The pioneering work is the code developed by Chodura and Schlüter [10]. In this code, an equilibrium is obtained by minimizing the potential energy with a friction method on cylindrical coordinates based on the variational principle. Hender et al. developed the NEAR code by improving the Chodura-Schlüter code with the employment of vacuum flux surfaces for the reference coordinates [11]. Recently, the PIES code [12] and the HINT code [13, 14] or the HINT2 code [15] have been developed and widely used for the stellarator equilibrium studies. Both codes are based on an iteration scheme of two steps. In the first step, the pressure profile consistent with the magnetic geometry with the magnetic field fixed. In the second step, the magnetic field satisfying the force balance equation is obtained with the pressure fixed. In the present work, we basically employ the two-step scheme.

The treatment in each step is different between the PIES and the HINT codes. In the first step of the PIES code, the pressure satisfying the equation of $\boldsymbol{B} \cdot \nabla P=0$ for a given magnetic field $\boldsymbol{B}$ is obtained with the field line tracing. In the second step, the plasma current is calculated by using this pressure $P$ and the field is obtained by solving the Ampére's law directly. Then, quasi magnetic coordinates are constructed with the field, and the next iteration is operated on the coordinates. The HINT code is based on the numerical scheme which was developed by Park et al. [13] for the reduced MHD equations, and is extended to the full MHD equations. Especially, the coordinate system twisted along the toroidal direction is employed for saving calculation regions. In the first step, the pressure satisfying $\boldsymbol{B} \cdot \nabla P=0$ is obtained as in the PIES code. The code by Park et al. and the original HINT code solve the equation by making the magnetic sound wave decay. The HINT2 code is improved so as to solve the equation $\boldsymbol{B} \cdot \nabla P=0$ directly by tracing the field lines. In the second step, a relaxation precess of the field is conducted with the equation of motion and the Faraday's law with $P$ fixed for obtaining the magnetic field satisfying the force balance condition.

Our numerical calculation method in this study is based on the concept of the code by Park and the HINT code. We treat MHD equilibria corresponding to the reduced MHD equations in a straight configuration and including a static magnetic island with a single helicity mode. Thus, we develop a more effective method than these codes. As described in Sec. 2, the cylindrical coordinates are utilized, and the Fourier expansion is employed in the poloidal and the toroidal directions. In the first step, a method utilizing a diffusion equation parallel to the magnetic field is employed, and an ordinary differential equation is solved in the second step. Therefore, the practical procedure is different from that of the HINT and the HINT2 codes. On the other hand, in general, not only the pressure diffusion parallel to the field, but also the diffusion perpendicular to field can affect the equilibrium pressure profile. Thus, we develop the code so as to include the diffusion perpendicular to the field in the first step.

This paper is organized as follows. The two-step approach for the equilibrium calculation is explained in Sec. 2. The equilibrium equations and the incorporation of the static islands are also described. In Sec. 3, the resultant equilibrium pressure is shown and the properties are discussed. In Sec. 4, the equilibrium including the diffusion perpendicular to the field is discussed. Concluding Remarks are given in Sec. 5.

\section{Two-Step Approach for Equilib- rium Calculation}

Here the numerical scheme to obtain MHD equilibria including static magnetic islands for the reduced MHD equations in a straight heliotron configuration is described. In a straight heliotron configuration, the normalized reduced MHD equations for the poloidal flux $\Psi$, the stream function $\Phi$ and the plasma pressure $P$ in the cylindrical coordinates $(r, \theta, z)$ are given by the Ohm's law,

$$
\frac{\partial \Psi}{\partial t}=-\boldsymbol{B} \cdot \nabla \Phi
$$

the vorticity equation,

$$
\frac{\mathrm{d} U}{\mathrm{~d} t}=-\boldsymbol{B} \cdot \nabla J_{z}+\frac{1}{2 \epsilon^{2}} \nabla \Omega \times \nabla P \cdot z,
$$

and the plasma pressure equation,

$$
\frac{\mathrm{d} P}{\mathrm{~d} t}=\kappa_{\perp} \nabla_{\perp}^{2} P+\kappa_{\|}(\boldsymbol{B} \cdot \nabla)(\boldsymbol{B} \cdot \nabla) P .
$$

The magnetic field $\boldsymbol{B}(r, \theta, z)$ is expressed as

$$
\boldsymbol{B}(r, \theta, z)=z+z \times \nabla \Psi(r, \theta, z)
$$

where $z$ denotes the unit vector in the $z$ direction. Here, $U(r, \theta, z)$ and $J z(r, \theta, z)$ are the vorticity in the negative $z$ direction and the current density in the $z$ direction, respectively. They are defined as

$$
U=\nabla_{\perp}^{2} \Phi \quad \text { and } \quad J z=\nabla_{\perp}^{2} \Psi
$$


where $\nabla_{\perp}^{2}$ is written as

$$
\nabla_{\perp}^{2}=\frac{1}{r} \frac{\partial}{\partial r} r \frac{\partial}{\partial r}+\frac{1}{r^{2}} \frac{\partial^{2}}{\partial \theta^{2}}
$$

The convective time derivative is given by

$$
\frac{\mathrm{d}}{\mathrm{d} t}=\frac{\partial}{\partial t}+\nabla \Phi \times z \cdot \nabla
$$

The quantity $\nabla \Omega$ denotes the averaged field line curvature. The factors $\epsilon, \beta_{0}, \kappa_{\perp}$ and $\kappa_{\|}$are the aspect ratio, the beta value at the magnetic axis, the perpendicular and the parallel pressure diffusion coefficients, respectively.

The quantities $\left(r, z, t, \Psi, \Phi, P, U, J z, \kappa_{\perp}, \kappa_{\|}\right)$are normalized by $\left(a, R_{0}, \tau_{\mathrm{A}}, a^{2} B_{0} / R_{0}, a^{2} / \tau_{\mathrm{A}}, B_{0}^{2} / 2 \mu_{0}, 1 / \tau_{\mathrm{A}}\right.$, $B_{0} / \mu_{0} R_{0}, a^{2} / \tau_{\mathrm{A}}, R_{0}^{2} / \tau_{\mathrm{A}}$ ), respectively. Here, $B_{0}, a, 2 \pi R_{0}$ and $\mu_{0}$ denote the magnetic field at the magnetic axis, the plasma radius, the one period length in $z$ direction and the vacuum permeability, respectively. The Alfvén time $\tau_{\mathrm{A}}$ is given by $\tau_{\mathrm{A}}=R_{0} \sqrt{\mu_{0} \rho} / B_{0}$, where $\rho$ is the mass density.

The equilibrium corresponding to Eqs. (1) (3) has to satisfy the condition that the pressure is constant along a field line, which is given by

$$
\boldsymbol{B} \cdot \nabla P=0,
$$

in arbitrary topology and the force balance equation,

$$
-\boldsymbol{B} \cdot \nabla J_{z}+\frac{1}{2 \epsilon^{2}} \nabla \Omega \times \nabla P \cdot z=0 .
$$

Equations (8) and (9) are coupled equations for $\Psi(r, \theta, z)$ and $P(r, \theta, z)$ to be solved. We express $P(r, \theta, z)$ and $\Psi(r, \theta, z)$ as the sum of the cylindrical symmetry component and other components as follows:

$$
\begin{aligned}
& P(r, \theta, z)=P_{\mathrm{sym}}(r)+\tilde{P}(r, \theta, z) \\
& \Psi(r, \theta, z)=\Psi_{\mathrm{sym}}(r)+\Psi_{m, n}^{\mathrm{ext}}(r, \theta, z)+\tilde{\Psi}(r, \theta, z)
\end{aligned}
$$

Here, $P_{\text {sym }}(r)$ and $\Psi_{\text {sym }}(r)$ denote the symmetry components and $\Psi_{m, n}^{\text {ext }}$ is the external poloidal flux corresponding to the external field, which generates static magnetic islands with the mode number of $(m, n)$. The tilde indicates the variation quantity of equilibrium due to the external field. In the case that the system is cylindrical symmetry without an external field, any functions of $r$ for $P_{\text {sym }}(r)$ and $\Psi_{\text {sym }}(r)$ are the solutions of Eqs. (8) and (9).

It is useful to express $\tilde{P}, \tilde{\Psi}, \Psi_{m, n}^{\text {ext }}$ with the Fourier series to solve Eqs. (8) and (9) as follows:

$$
\begin{aligned}
& \tilde{P}(r, \theta, z)=\sum_{m, n} \tilde{P}_{m, n}, \quad \tilde{P}_{m, n}=\hat{P}_{m, n}(r) \cos (m \theta-n z), \\
& \tilde{\Psi}(r, \theta, z)=\sum_{m, n} \tilde{\Psi}_{m, n}, \quad \tilde{\Psi}_{m, n}=\hat{\Psi}_{m, n}(r) \cos (m \theta-n z), \\
& \Psi_{m, n}^{\mathrm{ext}}(r, \theta, z)=\hat{\Psi}_{m, n}^{\mathrm{ext}}(r) \cos (m \theta-n z),
\end{aligned}
$$

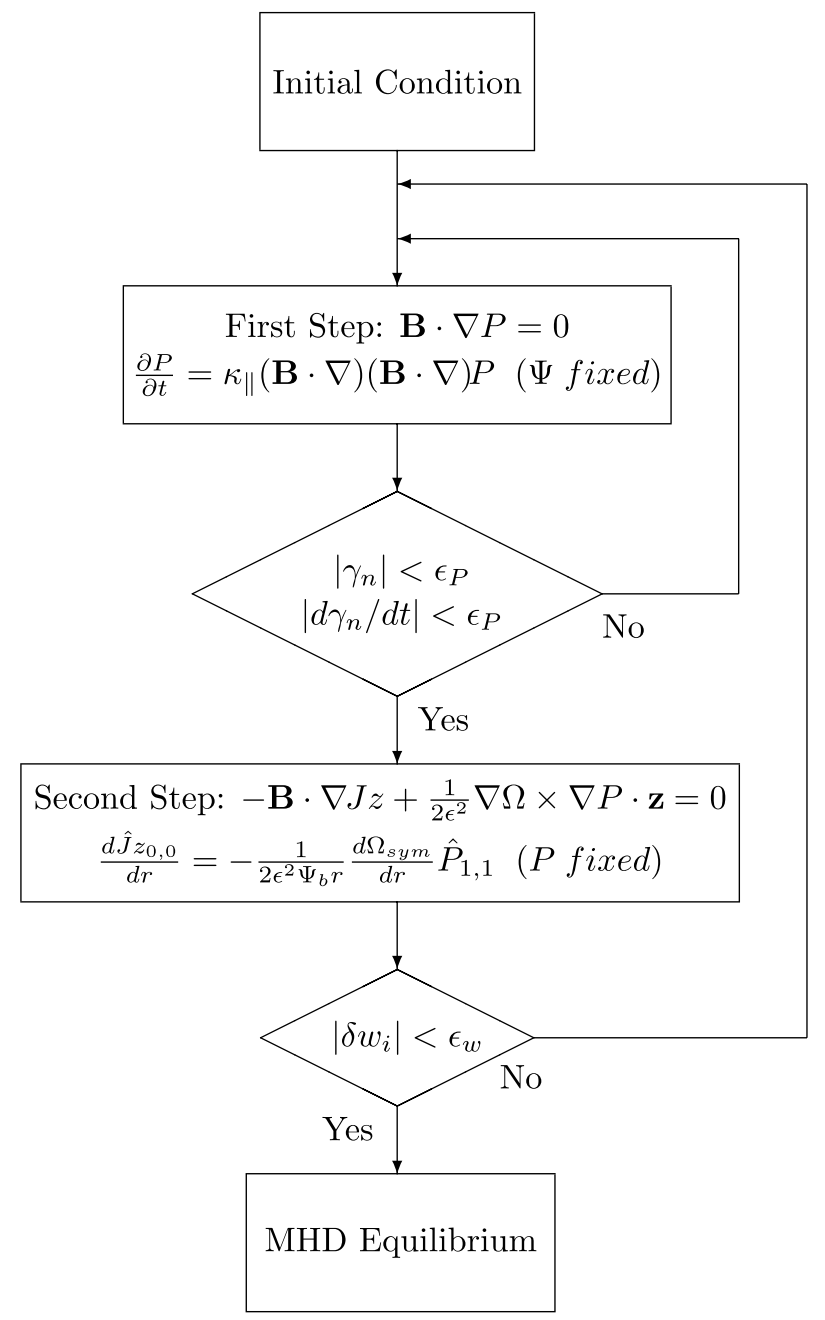

Fig. 1 Flow chart of the scheme.

where " $\wedge$ " means the Fourier coefficients. As is discussed in Refs. [7-9], $\hat{\Psi}_{m, n}^{\text {ext }}(r)$ is given by the solution of nocurrent condition,

$$
\nabla_{\perp}^{2} \Psi_{m, n}^{\mathrm{ext}}(r, \theta, z)=0
$$

under the boundary conditions of

$$
\hat{\Psi}_{m, n}^{\mathrm{ext}}(0)=0 \quad \text { and } \quad \hat{\Psi}_{m, n}^{\mathrm{ext}}(1)=\Psi_{\mathrm{b}} .
$$

Here, $\Psi_{\mathrm{b}}$ is the value of the external poloidal flux at the plasma boundary. In the case that there exists only the static magnetic island with a single mode, only the single helicity modes of $m / n=$ const. are sufficient in the expression of Eqs. (12) and (13) because of the helical symmetry. In this work, we treat the equilibrium including the static island with a single mode of $(m, n)=(1,1)$. In this case, $\Psi_{1,1}^{\text {ext }}$ generating the island is given by

$$
\Psi_{1,1}^{\mathrm{ext}}(r, \theta, z)=\Psi_{\mathrm{b}} r \cos (\theta-z),
$$

as the solution of Eq. (15).

In this paper, we solve Eqs. (8) and (9) in two separate steps as shown Fig. 1. These two steps are iterated until the 
MHD equilibrium is obtained. In the first step, $P$ satisfying Eq. (8) is obtained with $\Psi$ fixed. In order to solve Eq. (8), we employ a diffusion equation parallel to the magnetic field given by

$$
\frac{\partial P}{\partial t}=\kappa_{\|}(\boldsymbol{B} \cdot \nabla)(\boldsymbol{B} \cdot \nabla) P .
$$

The pressure $P$ constant along the field line is obtained when the stationary state of Eq. (18) is achieved. This equation is expanded in the Fourier series. In the present calculation, the modes in the range of $0 \leq n \leq N_{p}$ with $N_{p}=2$ are employed in Eq. (12). Then, $P$ is expressed as

$$
P(r, \theta, z)=P_{\text {sym }}(r)+\sum_{n=0}^{2} \hat{P}_{n, n}(r) \cos (n \theta-n z) .
$$

The Fourier component of Eq. (18) for each mode number of $n$ is written as follows:

$$
\begin{aligned}
\frac{\partial \hat{P}_{0,0}}{\partial t}= & \frac{\kappa_{\|} \Psi_{\mathrm{b}}}{2}\left[\frac{(1-\imath)}{r} \hat{P}_{1,1}+\frac{\Psi_{\mathrm{b}}}{r} \frac{\mathrm{d}}{\mathrm{d} r}\left(P_{\mathrm{sym}}+\hat{P}_{0,0}\right)\right. \\
& +(1-\imath) \frac{\mathrm{d} \hat{P}_{1,1}}{\mathrm{~d} r}-\frac{\mathrm{d} t}{\mathrm{~d} r} \hat{P}_{1,1} \\
& +\Psi_{\mathrm{b}} \frac{\mathrm{d}^{2}}{\mathrm{~d} r^{2}}\left(P_{\mathrm{sym}}+\hat{P}_{0,0}\right) \\
& \left.-\frac{\Psi_{\mathrm{b}}}{2}\left(\frac{\mathrm{d}^{2} \hat{P}_{2,2}}{\mathrm{~d} r^{2}}+\frac{3}{r} \frac{\mathrm{d} \hat{P}_{2,2}}{\mathrm{~d} r}\right)\right] \\
\frac{\partial \hat{P}_{1,1}}{\partial t}= & \kappa_{\|}\left\{-(1-\imath)\left[(1-\imath) \hat{P}_{1,1}\right.\right. \\
& \left.+\Psi_{\mathrm{b}} \frac{\mathrm{d}}{\mathrm{d} r}\left(P_{\mathrm{sym}}+\hat{P}_{0,0}\right)\right] \\
& +\frac{\Psi_{\mathrm{b}}^{2}}{4}\left(\frac{\mathrm{d}^{2} \hat{P}_{1,1}}{\mathrm{~d} r^{2}}+\frac{1}{r} \frac{\mathrm{d} \hat{P}_{1,1}}{\mathrm{~d} r}-\frac{1}{r^{2}} \hat{P}_{1,1}\right) \\
& +\frac{3 \Psi_{\mathrm{b}}}{2}(1-\imath) \frac{\mathrm{d} \hat{P}_{2,2}}{\mathrm{~d} r} \\
& \left.+3 \Psi_{\mathrm{b}}(1-\imath) \frac{\hat{P}_{2,2}}{r}-\Psi_{\mathrm{b}} \frac{\mathrm{d} t}{\mathrm{~d} r} \hat{P}_{2,2}\right\}
\end{aligned}
$$

and

$$
\begin{aligned}
\frac{\partial \hat{P}_{2,2}}{\partial t}= & \kappa_{\|}\left\{-2(1-\imath)\left[2(1-\imath) \hat{P}_{2,2}\right.\right. \\
& \left.+\frac{\Psi_{\mathrm{b}}}{2}\left(\frac{\mathrm{d} \hat{P}_{1,1}}{\mathrm{~d} r}-\frac{1}{r} \hat{P}_{1,1}\right)\right] \\
& +\frac{\Psi_{\mathrm{b}}}{2}\left[\frac{1-\imath}{r} \hat{P}_{1,1}+\frac{\Psi_{\mathrm{b}}}{r} \frac{\mathrm{d}}{\mathrm{d} r}\left(P_{\mathrm{sym}}+\hat{P}_{0,0}\right)\right. \\
& \left.+\frac{\mathrm{d} t}{\mathrm{~d} r} \hat{P}_{1,1}-(1-\imath) \frac{\mathrm{d} \hat{P}_{1,1}}{\mathrm{~d} r}\right] \\
& -\Psi_{\mathrm{b}} \frac{\mathrm{d}^{2}}{\mathrm{~d} r^{2}}\left(P_{\mathrm{sym}}+\hat{P}_{0,0}\right) \\
& \left.+\frac{\Psi_{\mathrm{b}}^{2}}{2}\left(\frac{\mathrm{d}^{2} \hat{P}_{2,2}}{\mathrm{~d} r^{2}}+\frac{1 \mathrm{~d} \hat{P}_{2,2}}{r}-\frac{4}{\mathrm{~d} r} \hat{P}_{2,2}\right)\right\} .
\end{aligned}
$$

Here, $t(r)$ is the rotational transform and is expressed with the components of $\Psi$ as

$$
t(r)=\iota_{\mathrm{sym}}(r)+\tilde{t}(r),
$$

where

$$
\iota_{\mathrm{sym}}(r)=\frac{1}{r} \frac{\mathrm{d} \Psi_{\mathrm{sym}}(r)}{\mathrm{d} r} \quad \text { and } \quad \tilde{\imath}(r)=\frac{1}{r} \frac{\mathrm{d} \hat{\Psi}_{0,0}(r)}{\mathrm{d} r}
$$

In order to judge the achievement of the steady state of Eq. (18), we define a parameter of $K_{n}$ for each mode number as

$$
K_{n}=\int_{0}^{1}\left\{\hat{P}_{n, n}(r)\right\}^{2} r \mathrm{~d} r
$$

We calculate the growth rate $\gamma_{n}$ given by

$$
\gamma_{n}=\frac{1}{K_{n}} \frac{\mathrm{d} K_{n}}{\mathrm{~d} t}
$$

and $\mathrm{d} \gamma_{n} / \mathrm{d} t$ every time step in the time evolution. When both conditions of

$$
\left|\gamma_{n}\right|<\epsilon_{p} \quad \text { and } \quad\left|\frac{\mathrm{d} \gamma_{n}}{\mathrm{~d} t}\right|<\epsilon_{p} \quad\left(\epsilon_{p} \ll 1\right),
$$

are satisfied simultaneously for each mode, we judge that the steady state is achieved.

In the second step, Eq. (9) is solved with $P$ fixed, which is obtained by the first step. To obtain the equilibrium including the static magnetic island with the single mode $(m, n)=(1,1)$, we employ the modes in the range of $0 \leq n \leq N_{\Psi}$ with $N_{\Psi}=1$ for $\Psi$. In this case, Eq. (11) is written as

$$
\begin{aligned}
\Psi(r, \theta, z)= & \Psi_{\mathrm{sym}}(r)+\Psi_{\mathrm{b}} r \cos (\theta-z) \\
& +\hat{\Psi}_{0,0}(r)+\hat{\Psi}_{1,1}(r) \cos (\theta-z)
\end{aligned}
$$

From Eq. (5), $J z$ is expressed as

$$
J z(r, \theta, z)=J_{z_{\mathrm{sym}}}(r)+\hat{J}_{z_{0,0}}(r)+\hat{J}_{z_{1,1}}(r) \cos (\theta-z) .
$$

In this study, no current condition for cylindrical equilibrium, $J z_{\text {sym }}=0$ is assumed. The averaged field line curvature $\mathrm{d} \Omega / \mathrm{d} r$ is fixed to $\mathrm{d} \Omega_{\mathrm{sym}} / \mathrm{d} r$, which is given by [16]

$$
\frac{\mathrm{d} \Omega_{\mathrm{sym}}}{\mathrm{d} r}=\frac{N_{t} \epsilon^{2}}{l} \frac{1}{r^{2}} \frac{\mathrm{d}}{\mathrm{d} r}\left(r^{4} \boldsymbol{t}_{\mathrm{sym}}\right),
$$

in the cylindrical geometry, where $N_{t}$ and $l$ are the toroidal period number and the pole number, respectively. We also expand Eq. (9) in the Fourier series. The $n=0$ component of Eq. (9) is satisfied trivially in this case. The $n=1$ component is written as

$$
\begin{aligned}
& -z \cdot \nabla \tilde{J}_{z_{1,1}}-\left[\Psi_{\mathrm{sym}}+\tilde{\Psi}_{0,0}, \tilde{J}_{z_{1,1}}\right] \\
& -\left[\Psi_{1,1}^{\mathrm{ext}}+\tilde{\Psi}_{1,1}, \tilde{J}_{z_{0,0}}\right]+\frac{1}{2 \epsilon^{2}}\left[\Omega_{\mathrm{sym}}, \tilde{P}_{1,1}\right]=0
\end{aligned}
$$

where $[f, g]$ is the Poisson bracket which is defined as

$$
[f, g]=\nabla f \times \nabla g \cdot z .
$$


Equation (31) has the solution of

$$
\tilde{\Psi}_{1,1}=\tilde{J}_{z_{1,1}}=0,
$$

and $\tilde{\Psi}_{0,0}$ satisfying

$$
\frac{\mathrm{d} \hat{J}_{z_{0,0}}}{\mathrm{~d} r}=-\frac{1}{2 \epsilon^{2} \Psi_{\mathrm{b}} r} \frac{\mathrm{d} \Omega_{\mathrm{sym}}}{\mathrm{d} r} \hat{P}_{1,1} .
$$

Thus, the force balance equation (9) is reduced to an ordinary differential equation for $\hat{\Psi}_{0,0}$. We obtain the solution for $\Psi$ by solving Eq. (34) for $\hat{\Psi}_{0,0}$ and substituting it into Eq. (28).

The width of the magnetic island $w_{i}$ is evaluated by using the solution of $\Psi$, where the subscript $i$ means the number of the iteration. The two steps described above are iterated until the width $w_{i}$ is converged. When the change rate $\delta w_{i}$ satisfies the condition,

$$
\left|\delta w_{i}\right|<\epsilon_{w} \quad\left(\epsilon_{w} \ll 1\right),
$$

we judge that the MHD equilibrium is obtained, where $\delta w_{i}$ is defined as

$$
\delta w_{i}=\frac{w_{i}-w_{i-1}}{w_{i-1}} .
$$

The island width is calculated from the shape of the magnetic island. The shape can be drawn by tracing the field line equations given by

$$
\frac{\mathrm{d} r}{\mathrm{~d} z}=\frac{B_{r}}{B_{z}}
$$

and

$$
\frac{\mathrm{d} \theta}{\mathrm{d} z}=\frac{B_{\theta}}{r B_{z}} .
$$

In the case of the helical symmetry with the mode numbers of $(m, n)$, the magnetic island shape can be drawn in an efficient way rather than tracing the Poincaré plots. We express the solution for Eqs. (37) and (38) as $(r(z), \theta(z))$ for the initial condition of $\left(r\left(z_{0}\right), \theta\left(z_{0}\right)\right)$ at $z=z_{0}$. In the change of the $z$ direction, the magnetic islands rotate $(m / n)\left(z-z_{0}\right)$ in the $\theta$ direction with keeping the shape. We can obtain the island shape at the cross section of $z=z_{0}$ by plotting the line of $\left(r(z), \theta(z)-(m / n)\left(z-z_{0}\right)\right)$. This procedure corresponds to subtracting the phase $(m / n)\left(z-z_{0}\right)$ in $\theta$ direction from the solution $\theta(z)$.

In the case of the magnetic island with $(m, n)$, there exist the X-point and the O-point at the positions where the right hand side of Eq. (38) equals to $m / n$. When the mode numbers are $(m, n)=(1,1)$, Eq. (38) combined with Eqs. (4), (24), (28) and (33) becomes

$$
\frac{\mathrm{d} \theta}{\mathrm{d} z}=\imath+\frac{\Psi_{\mathrm{b}}}{r} \cos (\theta-z) .
$$

In the case of positive $\Psi_{\mathrm{b}}$, there exist X-point at $\theta=0$ and $\mathrm{O}$-point at $\theta=\pi$ in the $z=0$ cross section. The radial coordinates $r$ 's for X-point and O-point satisfy

$$
t+\frac{\Psi_{\mathrm{b}}}{r}=1 \quad \text { and } \quad t-\frac{\Psi_{\mathrm{b}}}{r}=1,
$$

respectively. Therefore, the radial positions of X-point, Opoint and the rational surface with $t=1$ are different because of the finite value of $\Psi_{\mathrm{b}}$.

\section{Resultant Equilibrium}

By using the method explained in Sec. 2, we calculate the MHD equilibria including static magnetic islands in a straight heliotron plasma. We employ the magnetic configuration parameters of $N_{t}=10, l=2$ and $\epsilon=0.16$, which correspond to the LHD configuration. The pressure profile,

$$
P_{\text {sym }}(r)=\beta_{0}\left(1-r^{4}\right)^{2}, \quad \hat{P}_{n, n}(r)=0 \quad(0 \leq n \leq 2),
$$

with $\beta_{0}=0.16 \%$ is used for the initial condition. The profile of the initial rotational transform is shown in Fig. 2. The profile of $\Psi_{\text {sym }}$ is obtained by applying this profile to Eq. (24). The value of $\Psi_{\mathrm{b}}$ and the initial condition for $\hat{\Psi}_{m, n}$ are set to be $\Psi_{\mathrm{b}}=10^{-3}$ and $\hat{\Psi}_{m, n}=0$, respectively. We employ $\epsilon_{p}=5.0 \times 10^{-7}$ and $\epsilon_{w}=10^{-6}$ as the convergence parameters.

Figure 3 shows the time evolution of $K_{n},\left|\gamma_{n}\right|$ and $\left|\mathrm{d} \gamma_{n} / \mathrm{d} t\right|$ over the whole iteration. Dashed lines show the times when the steady state condition in the first step is achieved and the second step is conducted. It is found that the steady state of each component $\hat{P}_{n, n}$ is smoothly achieved for each iteration. Figure 4 shows $w_{i}$ and $\left|\delta w_{i}\right|$ at the times of the dashed lines in Fig. 3 as functions of the iteration number. As the iteration number increases, the island width becomes converged. The convergence condition is satisfied at $i=12$, and then, the equilibrium is obtained.

Figure 5 shows the profiles of the components of the equilibrium pressure $\hat{P}_{n, n}$. The component of $\hat{P}_{0,0}$ is dominant and $\hat{P}_{2,2}$ is much smaller than $\hat{P}_{1,1}$. The ratios of the maximum value of $\left|\hat{P}_{2,2}\right|$ to those of $\left|\hat{P}_{0,0}\right|$ and $\left|\hat{P}_{1,1}\right|$ are

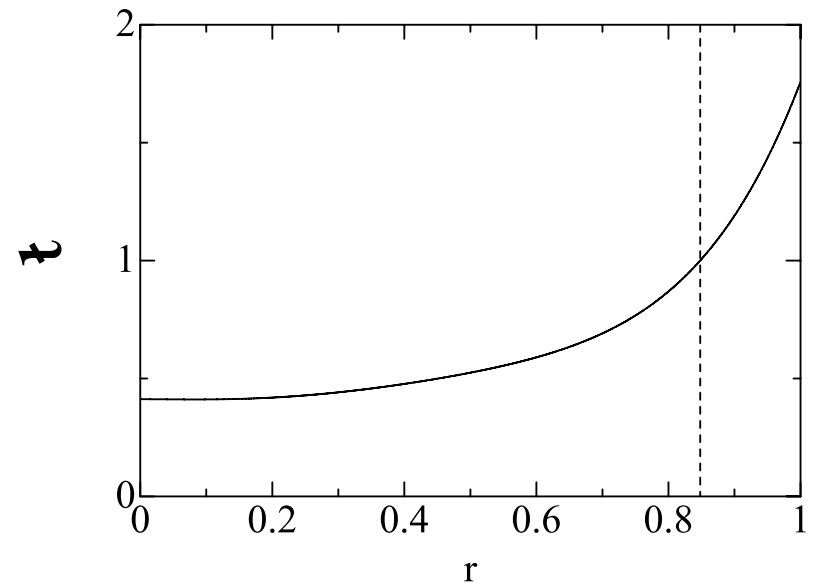

Fig. 2 Profile of initial rotational transform. Dashed line shows the position of the rational surface with $\imath=1$. 


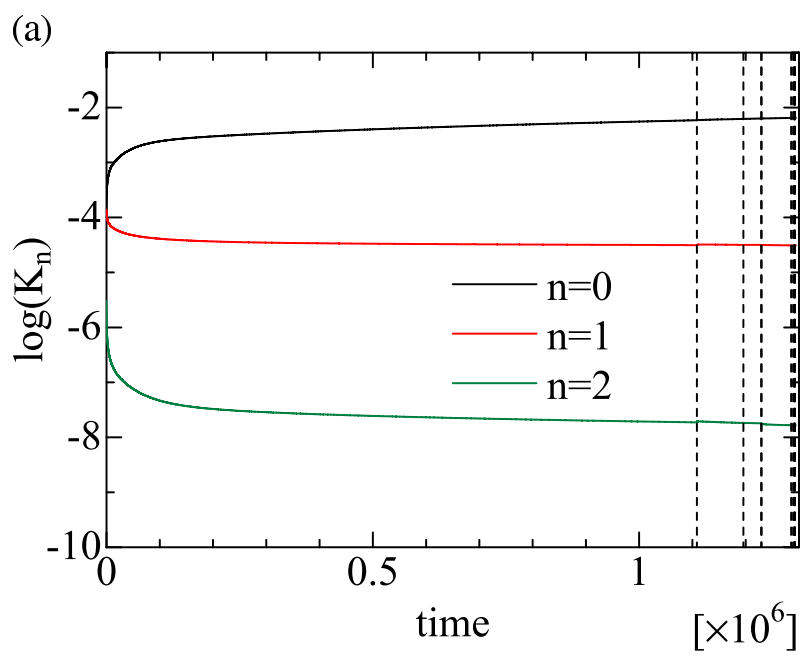

(b)

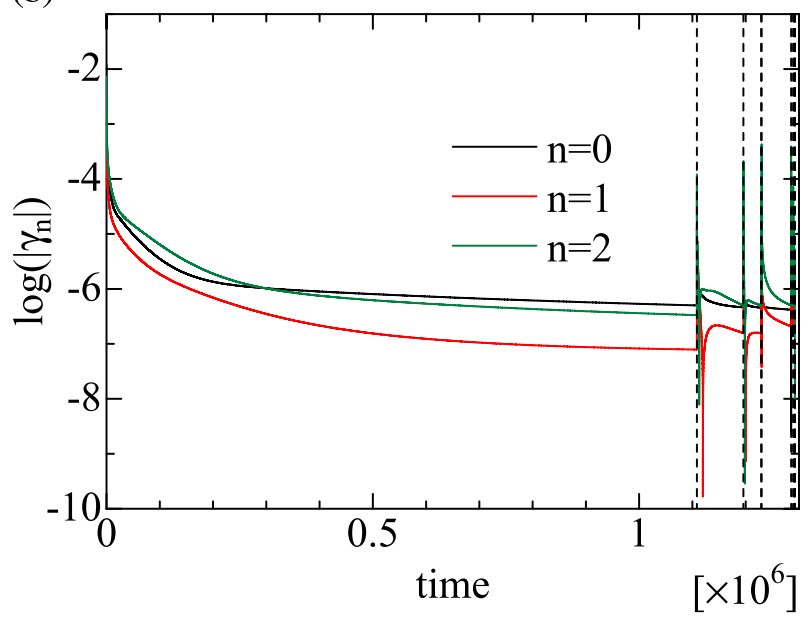

(c)

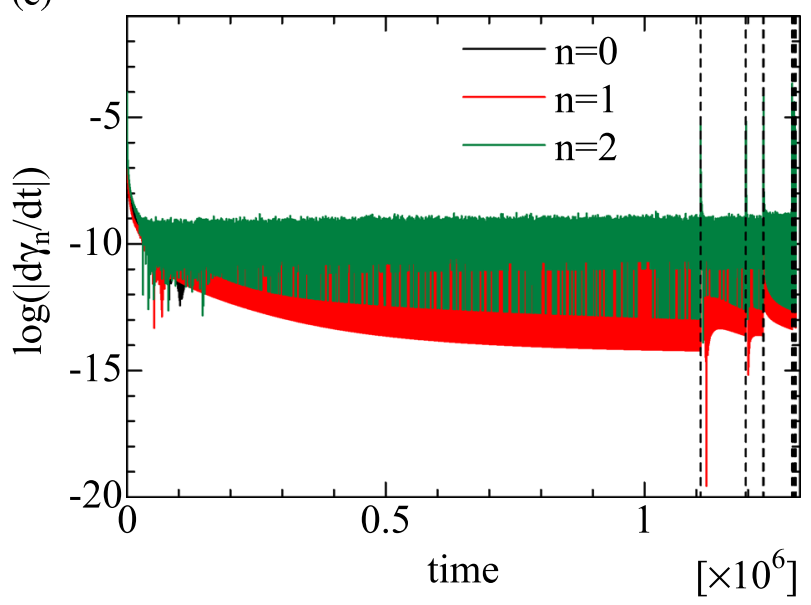

Fig. 3 Time evolution of (a) $K_{n}$, (b) $\left|\gamma_{n}\right|$ and (c) $\left|\mathrm{d} \gamma_{n} / \mathrm{d} t\right|$ for $\kappa_{\perp} / \kappa_{\|}=0$. Dashed lines indicate the times when the steady state condition is satisfied and the second step is conducted.

$\left|\hat{P}_{2,2}\right| /\left|\hat{P}_{0,0}\right|=7.1 \times 10^{-4}$ and $\left|\hat{P}_{2,2}\right| /\left|\hat{P}_{1,1}\right|=1.4 \times 10^{-2}$, respectively. This result confirms that $N_{p}=2$ is adequate in the first step calculation.

Figures 6 (a) and (b) show the contour of the constant pressure and the magnetic surfaces at $z=0$ cross (a)

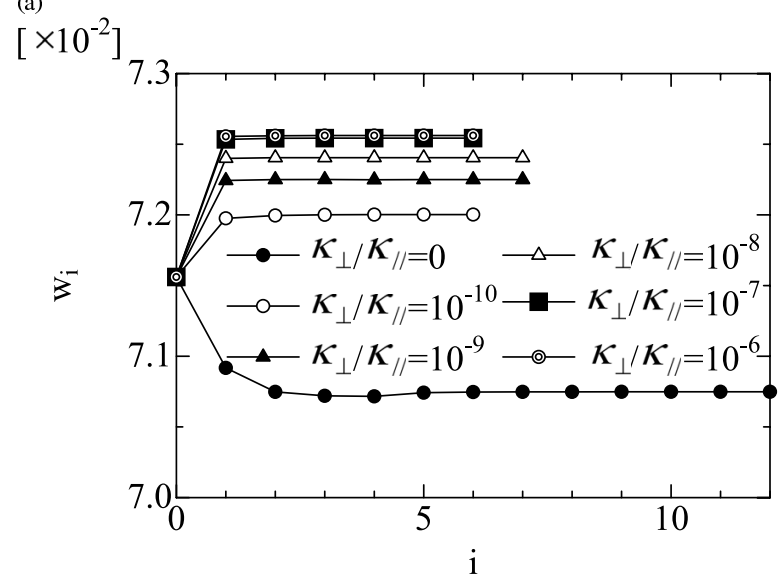

(b)

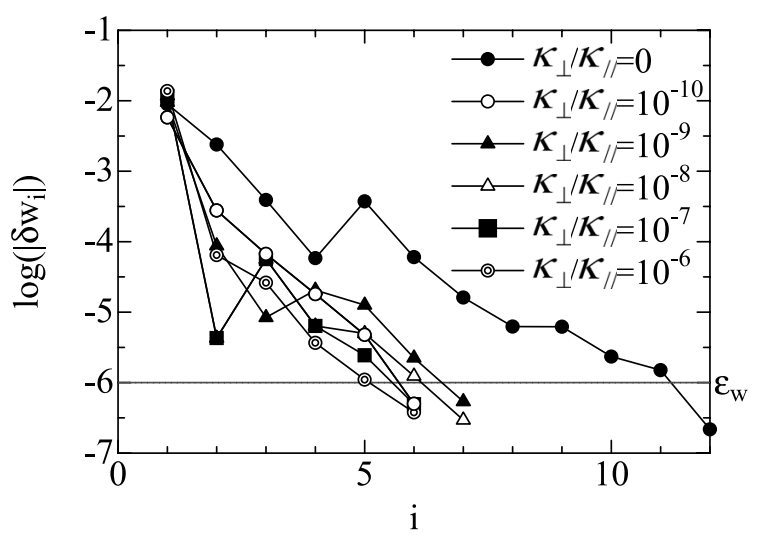

Fig. 4 Variation of (a) $w_{i}$ and (b) $\delta w_{i}$ for iteration number. The plots of $\kappa_{\perp} / \kappa_{\|}=0$ correspond to the case of Fig. 3 .

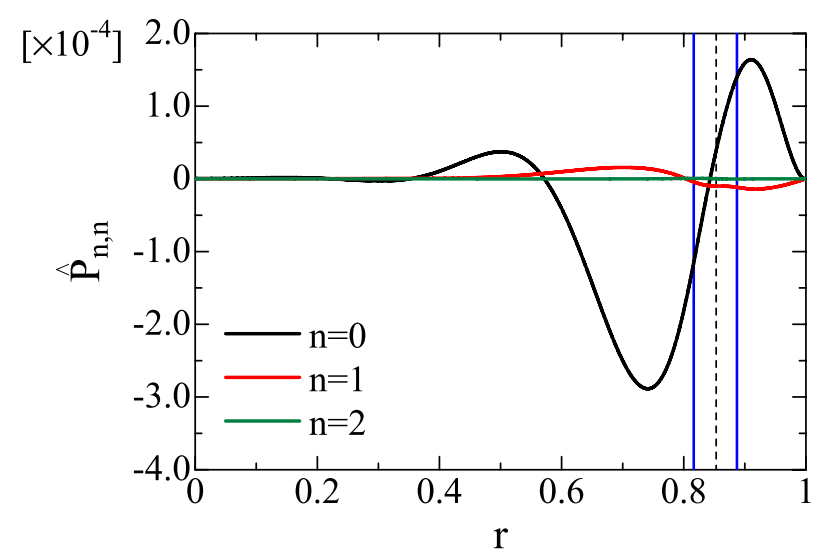

Fig. 5 Profiles of $\hat{P}_{n, n}$. Dashed lines indicate the position of the rational surface. Blue lines indicate the position of the separatrix of the island at $\theta=\pi$.

section in the resultant equilibrium. Since $\hat{P}_{2,2}$ is much smaller than $\hat{P}_{0,0}$ and $\hat{P}_{1,1}$ as described above, the contribution of $\hat{P}_{2,2}$ to the pressure contour is negligible. For this reason, we exclude $\tilde{P}_{2,2}$ in Eq. (19) when we plot the pressure contour in Fig. 6 (a). Figures 6 (a) and (b) show a good agreement between the pressure contour and the magnetic surfaces. The separatrix exists also in the pressure con- 
(a)

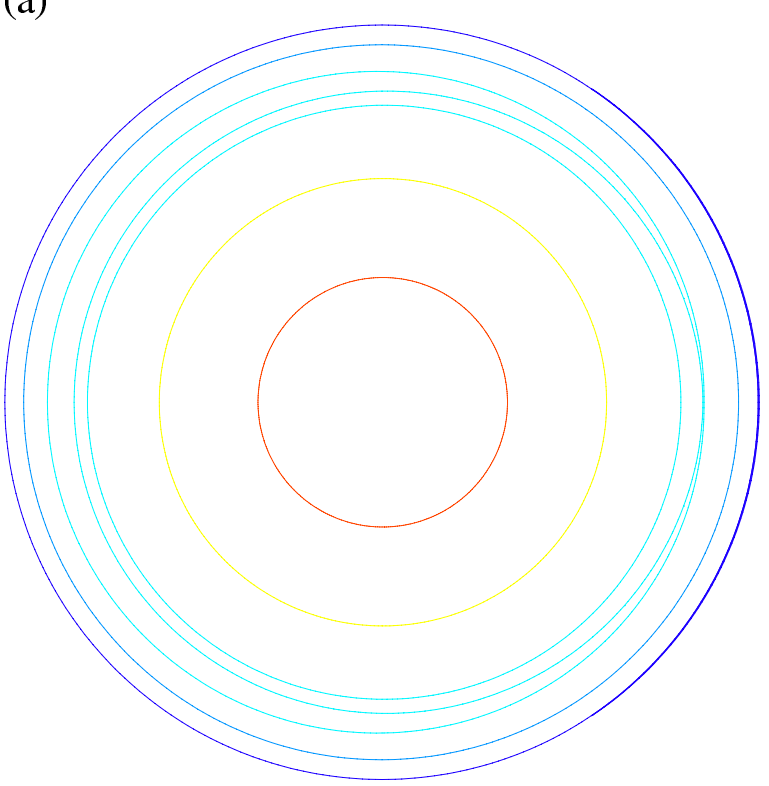

(b)

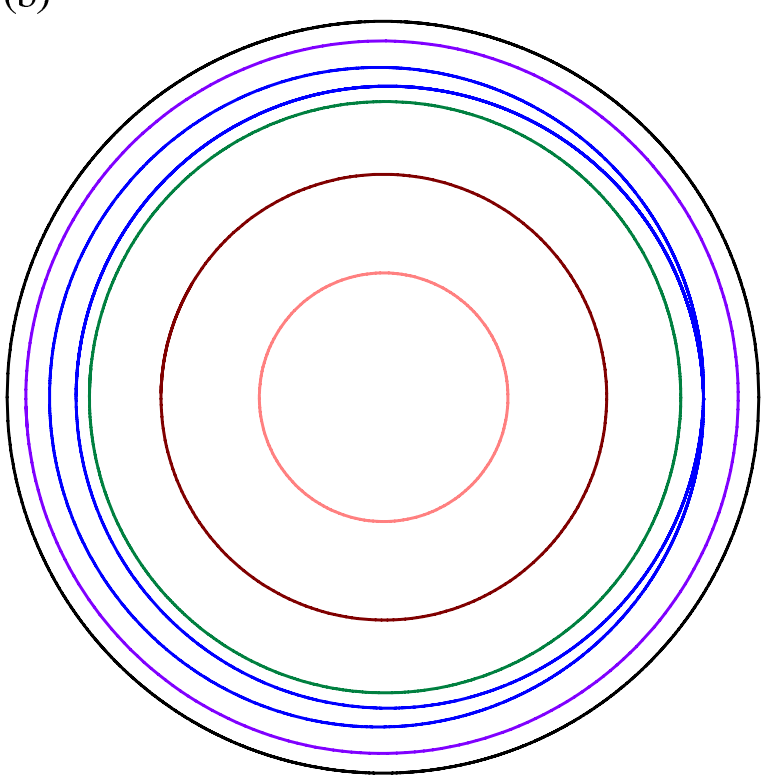

(c)

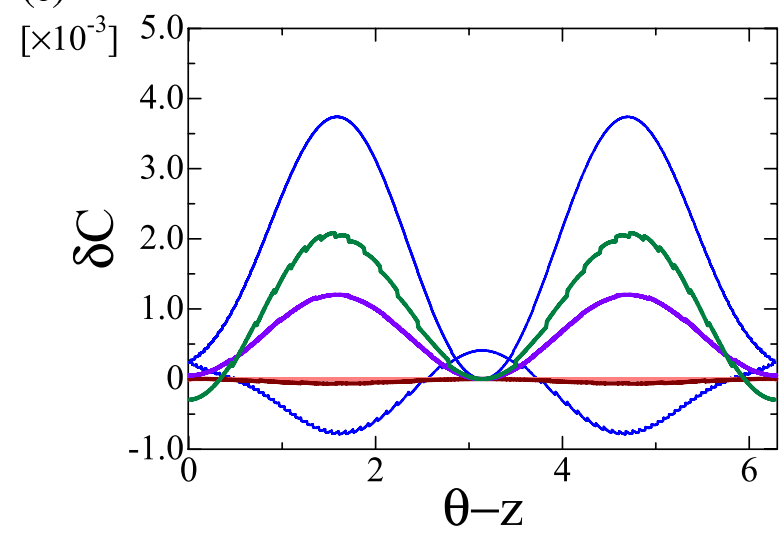

Fig. 6 Plots of (a) pressure contour and (b) magnetic surfaces of the resultant equilibrium at $\beta_{0}=0.16 \%$ on $z=0$ cross section and (c) relative error of pressure along field lines. Each field line in (c) is on the surface with the same color in (b). tour which corresponds to that of the magnetic island. Figure 6 (c) shows the relative error $\delta C$ in the pressure along the field line. The definition of $\delta C$ is given by

$$
\delta C(r, \theta-z)=\frac{P(r, \theta-z)-P\left(r_{0}, \theta_{0}-z_{0}\right)}{P\left(r_{0}, \theta_{0}-z_{0}\right)} .
$$

The relative error is evaluated along the field line of the total pressure without the component of $n=2$. Since the phase angle $\theta-z$ varies along the field line, we evaluate $\delta C$ as a function of $\theta-z$. The subscript 0 means the coordinates of the starting point of the field line. In Fig. 6 (c), $\theta_{0}=\pi$ and $z_{0}=0$ are used. The field lines corresponding to $r_{0}=0.329,0.591,0.781,0.816$ and 0.950 are chosen here. For fixed values of $\theta_{0}$ and $z_{0}$, the radial coordinate $r_{0}$ identifies the magnetic surface on which the field line is traced. The field lines of $r_{0}=0.329,0.591$ and 0.781 are on the magnetic surfaces inward of the separatrix (red, brown and green), $r_{0}=0.816$ the separatrix (blue) and $r_{0}=0.950$ the surface outward of the separatrix (purple), respectively. Each line in Fig. 6 (c) corresponds to the magnetic surface with the same color in Fig. 6(b). Figure 6 (c) shows that the largest error of the pressure appears on the field line of the separatrix (blue). Even in the case, however, the value is quite small and less than $5.0 \times 10^{-3}$. Therefore, Eq. (8) is satisfied in a good accuracy.

Figure 7 (a) shows the equilibrium pressure profile along the line connecting $(r=1, \theta=0, z=0)$ and $(r=1, \theta=\pi, z=0)$. Figures 7 (b) and (c) are the enlarged figures around the magnetic island at $\theta=0$ and $\theta=\pi$, respectively. The pressure profiles of both cases with and without $\tilde{P}_{2,2}$ are plotted in Fig. 7. However, the difference between the cases is too small to be distinguished because $\left|\hat{P}_{2,2}\right|$ is much less than the others. As shown in Fig. 7, the pressure profile is flat not only at the O-point but also at the $\mathrm{X}$-point. This property is explained with the expression of the Fourier component of $\boldsymbol{B} \cdot \nabla P$. In the equilibrium, each Fourier coefficient of $\boldsymbol{B} \cdot \nabla P$ is zero. The $(m, n)=(1,1)$ coefficient is given by

$$
(\boldsymbol{B} \cdot \nabla P)_{1,1}=(1-\imath) \hat{P}_{1,1}+\Psi_{\mathrm{b}} \frac{\mathrm{d}}{\mathrm{d} r}\left(P_{\mathrm{sym}}+\hat{P}_{0,0}\right),
$$

under the condition of $\hat{P}_{n, n}=0$ for $n \geq 2$. Since the first term equals to zero at the $t=1$ surface, the relation of $\mathrm{d} / \mathrm{d} r\left(P_{\text {sym }}+\hat{P}_{0,0}\right)=0$ must be satisfied. This equation indicates that the pressure profile is flat in the annular region near the surface involving both the O-point and the $\mathrm{X}$-point.

In Figs. 7 (b) and (c), the horizontal purple line shows the pressure value corresponding to the separatrix in the pressure contour shown in Fig. 6 (a). These figures show that the pressure has the same value at the $\mathrm{X}$-point and at the separatrix at $\theta=\pi$ of the magnetic surfaces. This result also confirms that the separatrix in the pressure contour coincides with the separatrix in the magnetic surfaces. 
(a)

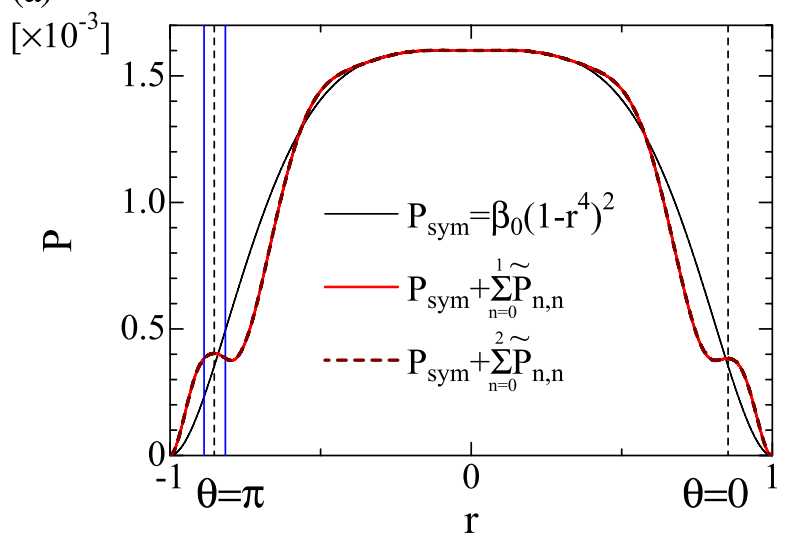

(b)

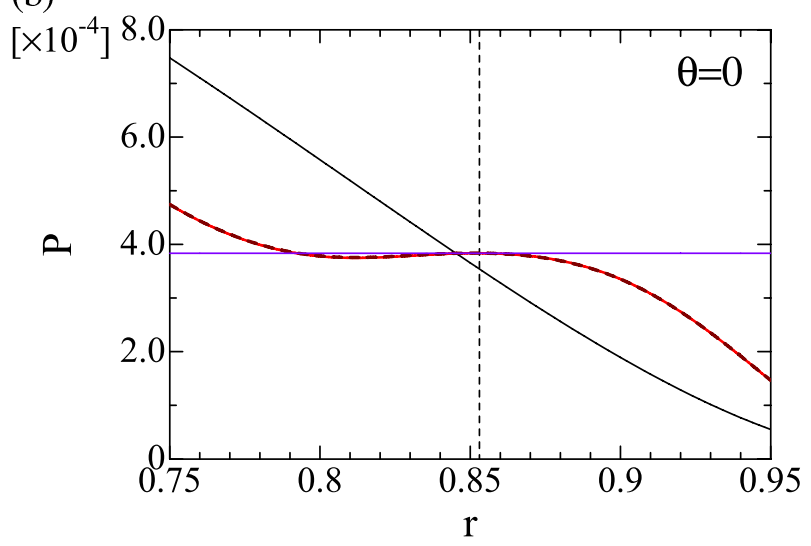

(c)

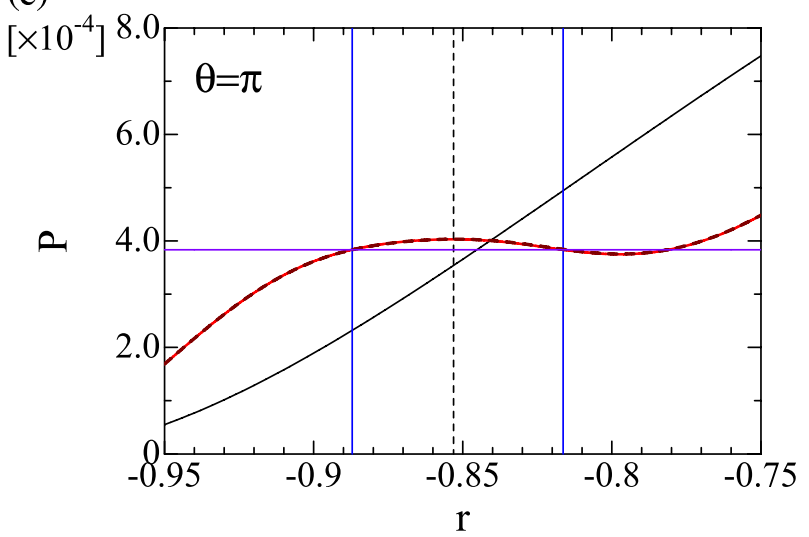

Fig. 7 Profile of resultant equilibrium pressure (a) along the line connecting $(r=1, \theta=0, z=0)$ and $(r=1, \theta=\pi, z=0)$ and its enlargements at (b) $\theta=0$ and (c) $\theta=\pi$. Red solid line and brown dashed line show the profiles of $P_{\text {sym }}+\sum_{n=0}^{1} \tilde{P}_{n, n}$ and $P_{\text {sym }}+\sum_{n=0}^{2} \tilde{P}_{n, n}$, respectively. Black line shows the profile of $P_{\text {sym }}$. Vertical dashed lines indicate the position of the rational surface. Blue lines indicate the positions of the separatrix of the magnetic island at $\theta=\pi$. Horizontal purple lines indicate the value of pressure at the separatrix in the pressure contour surfaces in Fig. 6 (a), $P=3.942 \times 10^{-4}$.

\section{Pressure Profile with Perpendicu- lar Heat Conductivity}

The effect of the pressure diffusion perpendicular to the field on the equilibrium pressure is also studied. In the magnetically confined plasma with a pressure gradient, the pressure is diffused following Eq. (3). The time scale of the parallel diffusion is much shorter than that of the perpendicular diffusion. Therefore, in the steady state, a static MHD equilibrium corresponding to the condition $\boldsymbol{B} \cdot \nabla P=0$ is considered to be achieved in a short time as the lowest approximation. As the next approximation, we consider a steady state including the perpendicular diffusion, which can be obtained in the transport time scale in the order of $10 \mathrm{msec} \sim 100 \mathrm{msec}$. In the steady state, a flow consistent with the diffusion should be taken into account. However, the incorporation of such a flow is quite complicated because the flow is determined not only by the pressure equation but also other transport equations. Thus, we assume the steady state with no flow for the approximation. Furthermore, for the steady state including the perpendicular diffusion, a heat source $S_{P}$ is needed in the pressure equation to compensate the decay of the total pressure. Here we also assume the source term corresponding to the form of $S_{P}=-\nabla_{\perp}^{2} P_{\text {sym }}$ in the pressure equation. Then, the resultant equation is given by

$$
\kappa_{\perp} \nabla_{\perp}^{2} \tilde{P}+\kappa_{\|}(\boldsymbol{B} \cdot \nabla)(\boldsymbol{B} \cdot \nabla) P=0 .
$$

By solving this equation together with Eq. (9), we can obtain a steady state with the perpendicular pressure diffusion with no flow including a static magnetic island. The solution is not an MHD equilibrium because the condition of $\boldsymbol{B} \cdot \nabla P=0$ is not satisfied. However, we can obtain the contribution of the perpendicular diffusion on the MHD equilibrium from the solution. To solve the equations, we employ the diffusion equation given by

$$
\frac{\partial P}{\partial t}=\kappa_{\perp} \nabla_{\perp}^{2} \tilde{P}+\kappa_{\|}(\boldsymbol{B} \cdot \nabla)(\boldsymbol{B} \cdot \nabla) P,
$$

instead of Eq. (18) for the first step and utilize the same scheme as in Sec. 2 for the second step.

Figure 8 shows the time evolution of $K_{n},\left|\gamma_{n}\right|$ and $\left|\mathrm{d} \gamma_{n} / \mathrm{d} t\right|$ for overall iterations in the case with $\kappa_{\perp} / \kappa_{\|}=10^{-7}$. In the first step, the steady state of Eq. (45) is obtained as in the case of $\kappa_{\perp} / \kappa_{\|}=0$. As shown in Fig. 4 (b), the convergence for the island width is also obtained in the result of the two-step iteration. In the case of $\kappa_{\perp} / \kappa_{\|}=10^{-7}$, the convergence is obtained at $i=6$. Similar time evolutions are obtained in the cases with $\kappa_{\perp} / \kappa_{\|}=10^{-6}, 10^{-8}, 10^{-9}$ and $10^{-10}$ as shown in Fig. 4(a). The island width in the resultant state decreases compared with the vacuum width in the case of $\kappa_{\perp} / \kappa_{\|}=0$ while the island widths increase in the cases of $\kappa_{\perp} / \kappa_{\|} \neq 0$. Figure 9 shows the dependence of the resultant pressure profile along the line connecting $(r=1, \theta=0, z=0)$ and $(r=1, \theta=\pi, z=0)$ on $\kappa_{\perp} / \kappa_{\|}$. As $\kappa_{\perp} / \kappa_{\|}$increases, the pressure gradient at the $\mathrm{X}$-point 


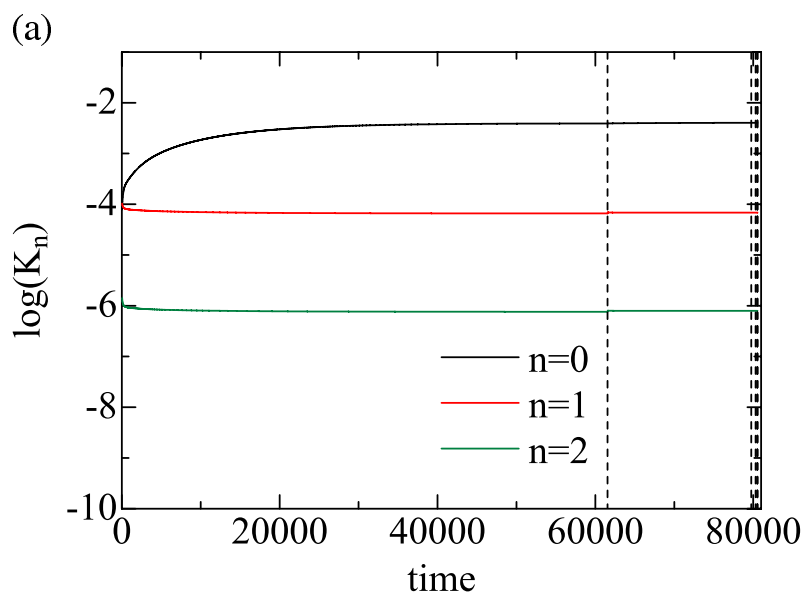

(b)

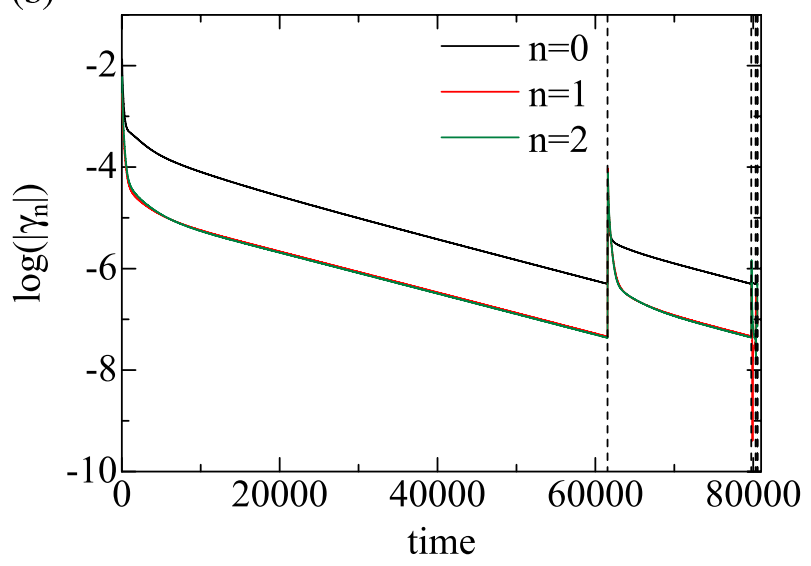

(c)

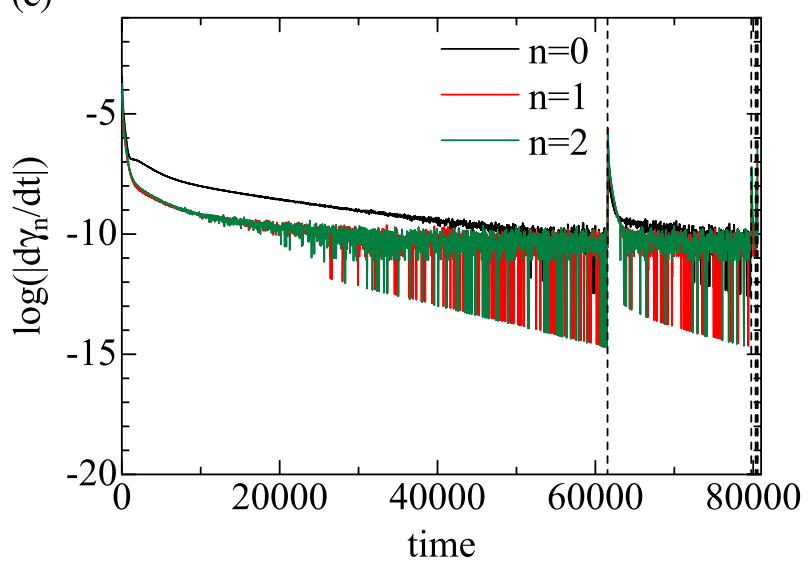

Fig. 8 Time evolution of (a) $K_{n}$, (b) $\left|\gamma_{n}\right|$ and (c) $\left|\mathrm{d} \gamma_{n} / \mathrm{d} t\right|$ for $\kappa_{\perp} / \kappa_{\|}=10^{-7}$. Dashed lines indicate the times when the steady state condition is satisfied and the second step is conducted.

enhances. In the case with $\kappa_{\perp} / \kappa_{\|}=10^{-7}$, the flat region almost disappears at the $\mathrm{X}$-point. The local flat structure at the $\mathrm{O}$-point is maintained for the finite value of $\kappa_{\perp} / \kappa_{\|}$, however, the width of the flat region decreases with the increase of $\kappa_{\perp} / \kappa_{\|}$.

In spite of that we do not take the diffusion for $P_{\text {sym }}(r)$ into account, the reduction of $P(r=0)$ in the resultant state is seen in Fig. 9 (a). This is due to the fact that $\hat{P}_{0,0}$ has a (a)

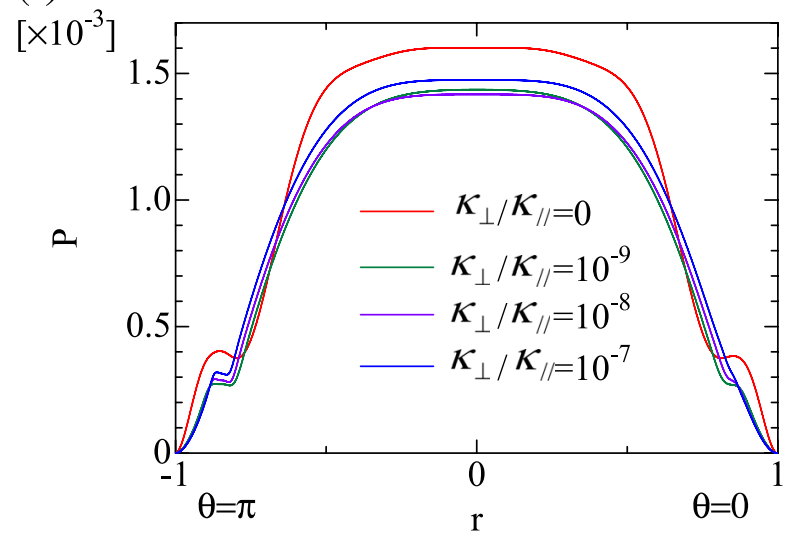

(b)

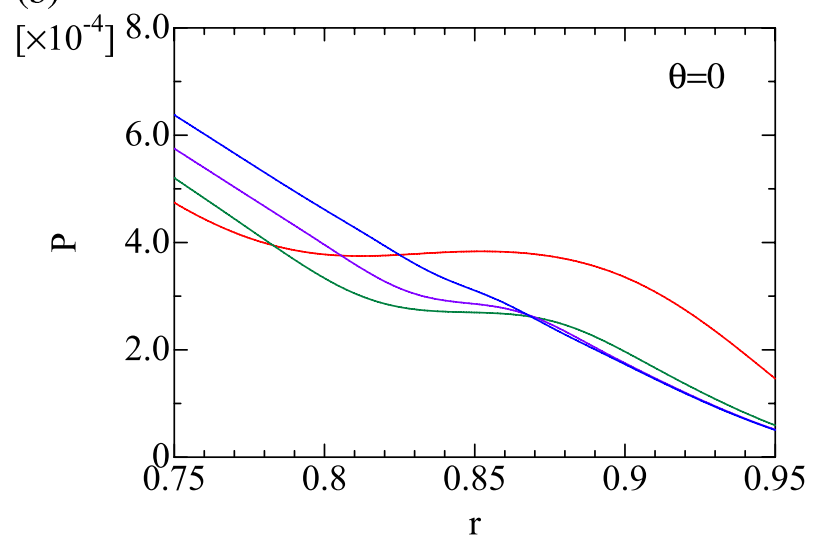

(c)

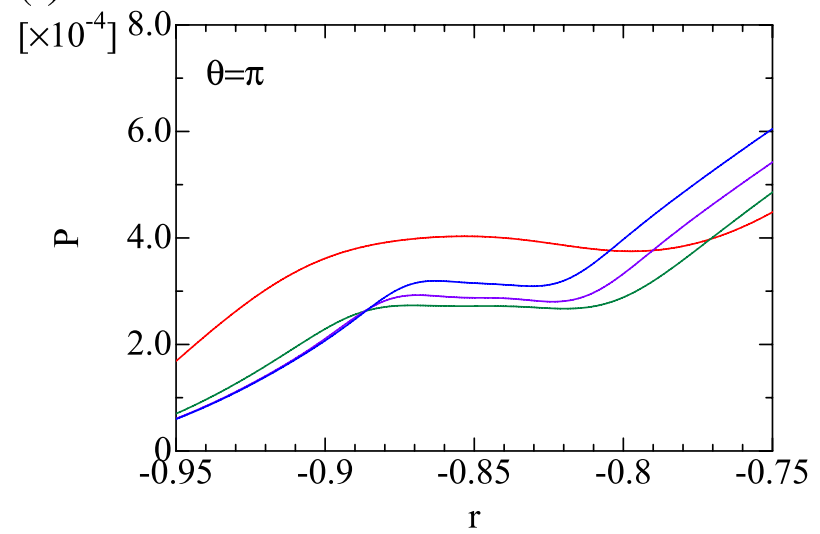

Fig. 9 Profiles of resultant pressure (a) along the line connecting $(r=1, \theta=0, z=0)$ and $(r=1, \theta=\pi, z=0)$ and its enlargements at (b) $\theta=0$ and (c) $\theta=\pi$ for $\kappa_{\perp} / \kappa_{\|}=0$, $10^{-9}, 10^{-8}$ and $10^{-7}$.

negative finite value at $r=0$ generated by the diffusion perpendicular to the field as shown in Fig. 10. Two kinds of contribution of the perpendicular diffusion on $\hat{P}_{0,0}$ around the rational surface and the magnetic axis lead to the negative value of $\hat{P}_{0,0}(r=0)$. As shown in Fig. 5, the profile of $\hat{P}_{0,0}$ locally has a negative value region just inside the rational surface in the case of $\kappa_{\perp} / \kappa_{\|}=0$. In the case of finite $\kappa_{\perp} / \kappa_{\|}$, the radial diffusion term works so as to reduce the curvature of the profile. It is followed that the region with the negative $\hat{P}_{0,0}$ is enlarged. On the other hand, in 


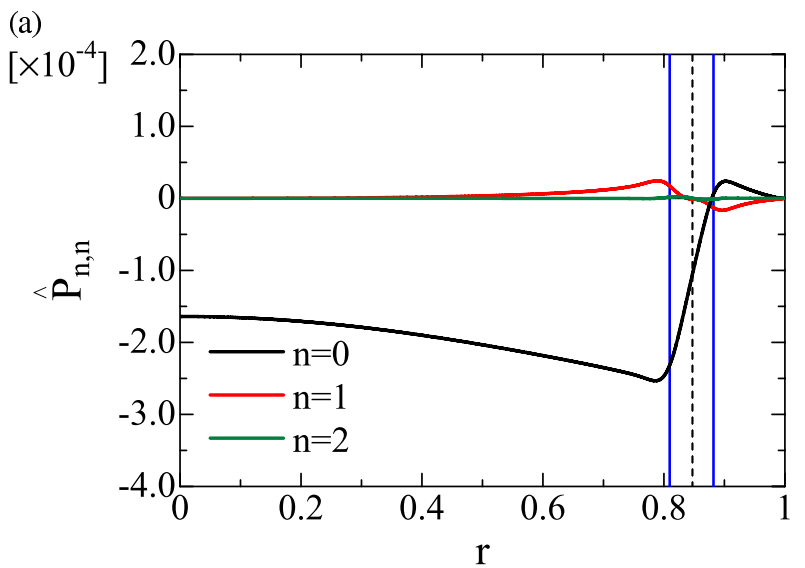

(b)

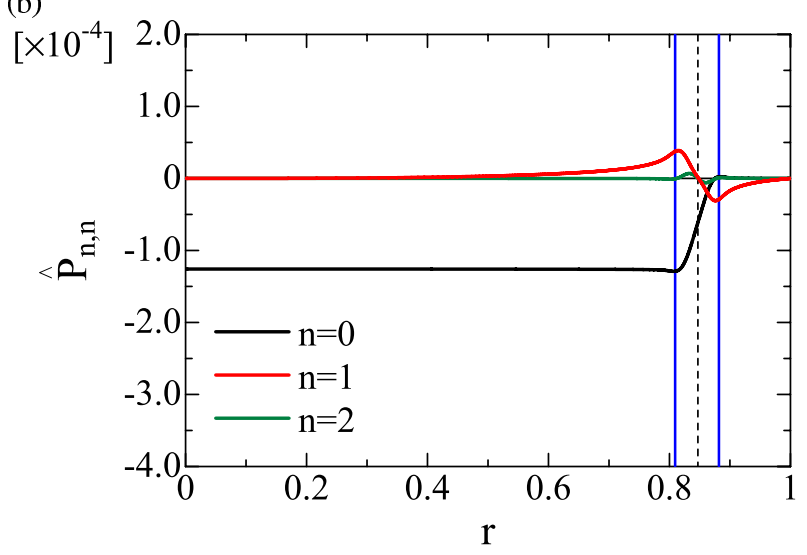

Fig. 10 Profiles of $\hat{P}_{n, n}$ for (a) $\kappa_{\perp} / \kappa_{\|}=10^{-9}$ and (b) $\kappa_{\perp} / \kappa_{\|}=10^{-7}$. Dashed lines indicate the position of the rational surface. Blue lines indicate the position of the separatrix of the island at $\theta=\pi$.

the region around the magnetic axis, $\hat{P}_{0,0}$ is almost zero in the case of $\kappa_{\perp} / \kappa_{\|}=0$ as shown in Fig. 5 , because the island effect is limited to the region around the rational surface. In the cases of finite $\kappa_{\perp} / \kappa_{\|}$, the first term is dominant in Eq. (44), and therefore, $\hat{P}_{0,0}$ satisfies the equation of

$$
\left(\nabla_{\perp}^{2} \tilde{P}\right)_{0,0}=\frac{\mathrm{d}^{2} \hat{P}_{0,0}}{\mathrm{~d}^{2} r}+\frac{1}{r} \frac{\mathrm{d} \hat{P}_{0,0}}{\mathrm{~d} r}=0,
$$

in this region. The solution of Eq. (46) which is regular at the axis is a constant. Therefore, the finite value of $\kappa_{\perp} / \kappa_{\|}$ makes the pressure profile constant around the axis. Since the solution of $\hat{P}_{0,0}$ has to be continuous between the regions around the rational surface and the axis, $\hat{P}_{0,0}$ has a finite and negative value at $r=0$ as shown in Fig. 10. That is, $P(r=0)$ is decreased in the cases with a finite value of $\kappa_{\perp} / \kappa_{\|}$. The absolute value of the decrease of $\hat{P}_{0,0}(r=0)$ does not change monotonously for the increase of $\kappa_{\perp} / \kappa_{\|}$as shown in Fig. 9 (a). This is because the two contributions are almost in a trade-off relation. In the increase of $\kappa_{\perp} / \kappa_{\|}$, the maximum absolute value of $\hat{P}_{0,0}$ around the rational surface is decreased because of the reduction of the curvature of $\hat{P}_{0,0}$ while the contribution making $\hat{P}_{0,0}$ constant is enhanced.

\section{Concluding Remarks}

An MHD equilibrium including a static magnetic island for the reduced MHD equations at $\beta_{0}=0.16 \%$ is obtained in a straight heliotron configuration. The equations to be solved are the coupled equations for the poloidal flux and the pressure. The equations are solved by iterating two numerical steps. In the formulation, the Fourier expansion is employed and the static island with $(m, n)=(1,1)$ is treated. In the first step, the equation of $\boldsymbol{B} \cdot \nabla P=0$ is solved with the poloidal flux fixed so that the pressure constant along the field line is obtained. In the present study, we utilize a diffusion equation parallel to the field line to solve this equation. The steady state solution of the diffusion equation corresponds to the pressure constant along the field line. Three Fourier components of $\hat{P}_{0,0}, \hat{P}_{1,1}$ and $\hat{P}_{2,2}$ are necessary at least to obtain the steady state. In the final equilibrium pressure, $\hat{P}_{2,2}$ is negligibly small compared with other components at $\beta_{0}=0.16 \%$, and therefore, it is not necessary in the second step. Nevertheless, $\hat{P}_{2,2}$ is needed for the sufficient steady state solution in the first step. In the second step, the force balance equation for the poloidal flux, which is derived from the vorticity equation, is solved with the pressure fixed. Since $\hat{P}_{2,2}$ and higher pressure components can be neglected, the Fourier series of the equation is truncated up to $n=1$. In this case, the condition of $\tilde{\Psi}_{1,1}=0$ and an ordinary differential equation for $\tilde{\Psi}_{0,0}$ are derived from the force balance equation. Therefore, only $\tilde{\Psi}_{0,0}$ is updated with the solution of the ordinary equation in the second step.

In the resultant equilibrium, we obtain a pressure profile which corresponds to the island structure. A separatrix is seen also in the pressure contour plot, however, the pressure gradient is zero at the rational surface. That is, local flattening appears at not only the O-point but also the Xpoint. Thus, the existence of the equilibrium with the pressure profile flat at the X-point is explicitly demonstrated in the present study. The clear identification of the X-point is required for the demonstration, which is possible only in the cylindrical geometry and the single helicity configuration employed in the present work, while the X-point is not clearly identified in three-dimensional equilibria due to the overlap of the multiple Fourier components in general. The equilibrium depends on the symmetry part of the pressure and the poloidal flux, $P_{\text {sym }}$ and $\Psi_{\text {sym }}$, which are used as the initial condition, even if the magnetic field is almost vacuum one.

It is noted that this scheme of the second step cannot be applied to higher beta cases as it is. At the low beta case such as $\beta_{0}=0.16 \%$, we obtain a satisfying accuracy in the calculation with only a small number of the Fourier series for $\tilde{\Psi}$ and $\tilde{P}$. This is attributed the fact that the solution of the magnetic field is close to the vacuum field. At higher beta, the deviation of the magnetic island shape from the vacuum one is enhanced, which degrades the accuracy of the approximation with the small number of the Fourier modes. Therefore, higher components are necessary in the 
second step for keeping the accuracy. In this case, the force balance equation becomes coupled equations for multiple number of $\tilde{\Psi}_{n, n}$, not a single ordinary differential equation.

On the other hand, we also examine the effect of the pressure diffusion perpendicular to the field line in the first step. As the perpendicular diffusion coefficient increases, the pressure gradient is enhanced at the X-point. A pressure profile flattened only at the O-point not the X-point can be obtained for a sufficiently large coefficient. The pressure at the axis is also affected by the perpendicular diffusion so as to be decreased through the change in the radial profile of $\tilde{P}_{0,0}$. The present result is obtained under the assumption of no flow steady state with a special type of heat source. Precise analysis with more realistic flow and heat source remains as a future work.

\section{Acknowledgments}

The authors would like to thank Dr. Y. Suzuki and Dr. R. Kanno for useful suggestions and lots of comments. This work is partly supported by the budget NIFS11KNXN222 of National Institute for Fusion Science and by Grant-in-Aid for Scientific Research (C) 22560822 from JSPS, Japan.

[1] A. Komori et al., Proc. 22th Fusion Energy Conf. OV/2-4 (2008).
[2] K. Ichiguchi, N. Nakajima, M. Wakatani, B.A. Carreras and V.E. Lynch, Nucl. Fusion 43, 1101 (2003).

[3] K. Ichiguchi and B.A. Carreras, J. Plasma Fusion Res. SERIES 6, 589 (2004).

[4] K. Saito, K. Ichiguchi and N. Ohyabu, Phys. Plasmas 17, 062504 (2010).

[5] K. Saito, K. Ichiguchi and R. Ishizaki, Plasma Fusion Res. 6, 2403072 (2011)

[6] H. Strauss, Plasma Phys. 22, 733 (1980).

[7] T. Unemura, S. Hamaguchi and M. Wakatani, Phys. Plasmas 11, 1545 (2004).

[8] L. Garcia, B.A. Carreras, V.E. Lynch, M.A. Pedrosa and C. Hidalgo, Phys. Plasmas 8, 4111 (2001).

[9] L. Garcia, B.A. Carreras, V.E. Lynch and M. Wakatani, Nucl. Fusion 43, 553 (2003).

[10] R. Chodura and A. Schlüter, J. Comput. Phys. 41, 68 (1981).

[11] T.C. Hender, B.A. Carreras, L. Garcia and J.A. Rome, J. Comput. Phys. 60, 76 (1985).

[12] A.H. Reiman and H. Greenside, Comput. Phys. Commun. 43, 157 (1986).

[13] W. Park, D.A. Monticello, H. Strauss and J. Manickam, Phys. Fluids 29 (4), 1171 (1986).

[14] K. Harafuji, T. Hayashi and T. Sato, J. Comput. Phys. 81, 169 (1989).

[15] Y. Suzuki, N. Nakajima, K.Y. Watanabe, Y. Nakamura and T. Hayashi, Nucl. Fusion 46, L19 (2006).

[16] M. Wakatani, Stellarator and Heliotron Devices (Oxford University Press, New York, 1998) p.98. 